\title{
A Multiline Multi-material Calibration Method for Liquid Characterization
}

\author{
Xiue Bao, Song Liu, Ilja Ocket, Member, IEEE, Juncheng Bao, Dries Kil, Meng Zhang, Bob Puers, Fellow, IEEE, \\ Dominique Schreurs, Fellow, IEEE, and Bart Nauwelaers, Senior Member, IEEE
}

\begin{abstract}
A multiline and multi-material calibration method is proposed, which is aimed at replacing state-of-the-art twotier microwave calibration techniques in liquid characterization process. The technique combines a general "line-line" trace method and an equivalent impedance algorithm, which enables to use unknown materials loaded on various lengths of transmission lines in the calibration standards. The validation measurements are performed on coplanar waveguide (CPW) structures due to the convenient fabrication and easy integration with polymers. Measurement comparison with the previous calibration approach verifies its capability in high frequency applications.
\end{abstract}

Index Terms - calibration, coplanar waveguide, microwave measurements, nonlinear least squares.

\section{INTRODUCTION}

Dielectric spectroscopy measurements can provide valuable information of various liquids and solutions for researches and studies in the biological and chemical fields. However, broadband quantitative characterization relies on accurate calibration of the vector network analyzer (VNA) and the test fixture of a device under test (DUT) . Differing in equipment by three or four samplers, a VNA can be calibrated with a twelve-term error model and an eight-term error model, respectively. With an addition of switch terms [1], the twelveterm problem can be solved with the eight-term algorithm, e.g. TRL ("through-reflect-line") [2] and LRM ("line-reflectmatch") [3]. Practically, only seven parameters are required to solve the eight-term problem [1], [4].

Though there are many accurate VNA calibration approaches [1]-[3], [5], they could not remove the unwanted effects of test fixtures where the DUT is embedded. To deembed errors caused by the test fixture, a second-tier calibration, or called "de-embedding" [6], is usually required. For instance, a reference liquid calibration method that allows any combination of fully or partially defined liquid standards has been used in water characterization [4], [7]. However, it still relies on accurate first-tier calibration.

Manuscript received January 14, 2018; revised May 2nd, 2018; accepted June 1st, 2018. This work was supported by the KU Leuven C2 ( $\left.\mu^{2} \mathrm{BIO}\right)$ and Hercules project funding. (Corresponding author: Xiue Bao.)

X. Bao, J. Bao, M. Zhang, D. Schreurs and B. Nauwelaers are with the Department of Electrical Engineering, KU Leuven, 3001 Leuven, Belgium (e-mail: xiue.bao@kuleuven.be).

S. Liu was with the Science and Technology on Metrology and Calibration Laboratory, Beijing Institute of Radio Metrology and Measurement, Beijing 100854, China.

I. Ocket is with the Interuniversity Microelectronics Center, 3001 Heverlee, Belgium, and also with the Department of Electrical Engineering, KU Leuven, 3001 Leuven, Belgium.

D. Kil and B. Puers are with the Div. ESAT-MICAS, KU Leuven, 3001, Leuven, Belgium.

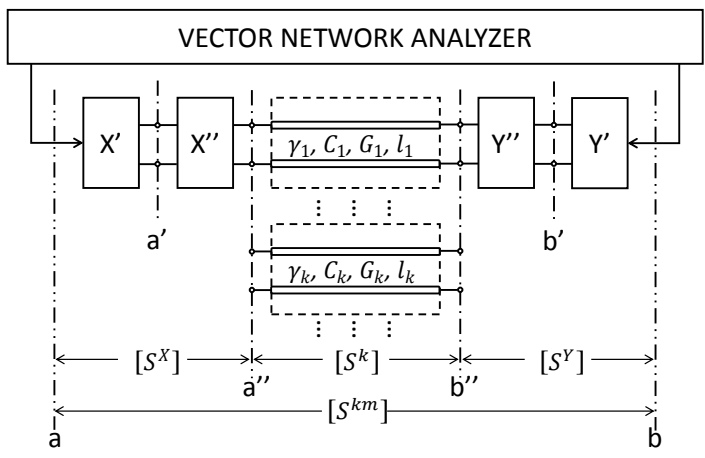

Fig. 1. Schematic of the measurement configuration, where $S^{X}$ and $S^{Y}$ are the two error boxes considered, $\gamma_{k}, C_{k}, G_{k}$, and $l_{k}$ are related to the $k$ th standard, and $S^{k m}$ is the measured S-parameters of the $k$ th standard.

In this study, instead of the two-step procedure, we develop a transmission line based multiline multi-material calibration method that can directly move the reference planes from VNA ports to the DUT. The transmission lines are required to be either clearly distinct in lengths or loaded with notably different dielectric materials. This paper is organized as follows: section II describes the proposed multiline multi-material calibration procedure; Section III introduces the on-wafer measurement setup and devices, presents the validation procedure, and also discusses the results; conclusions are drawn in the final section.

\section{Method DESCRIPTION}

Fig. 1 shows the configuration of a typical on-wafer measurement procedure [6]. $X^{\prime}$ and $Y^{\prime}$ represent the two error boxes caused by the connections and cables between VNA ports and probe-tips. $X^{\prime \prime}$ and $Y^{\prime \prime}$ are errors due to the test fixture from probe-tips to the DUT. $X^{\prime}$ and $Y^{\prime}$ are often removed with a first-tier calibration, followed by a secondtier calibration to remove $X^{\prime \prime}$ and $Y^{\prime \prime}$. In this study, the twotier calibration process is replaced with a one-tier calibration, with the scattering (S-)parameters $S^{X}$ resulting from $X^{\prime}$ and $X^{\prime \prime}$, and $S^{Y}$ resulting from $Y^{\prime}$ and $Y^{\prime \prime}$, respectively. To characterize $S^{X}$ and $S^{Y}$, seven parameters, i.e., $S_{12}^{X} S_{12}^{X}, S_{11}^{X}$, $S_{22}^{X}, S_{21}^{X} S_{12}^{Y}, S_{12}^{Y} S_{12}^{Y}, S_{11}^{Y}$, and $S_{22}^{Y}$, are required [1], [4].

The DUTs we use are identical coplanar waveguide (CPW) transmission lines fabricated on a low loss substrate and being of the same cross-sectional dimensions, on top of which various dielectric materials can be loaded. One line is loaded with a reference material of known permittivity, and another line is covered with an unknown dielectric material. The 
reference material line is $l_{r}$ long, and its propagation constant, distributed per unit length (p.u.l) capacitance, and p.u.l conductance [8] are denoted as $\gamma_{r}, C_{r}$, and $G_{r}$, respectively. The corresponding parameters of the line with an unknown material are $l_{t}, \gamma_{t}, C_{t}$, and $G_{t}$, respectively. As the p.u.l resistance $R$ and inductance $L$ are determined only by the electrodes' geometries and properties, they present the same values for all of the CPW lines covered with different materials [9]. Therefore, according to the equivalent impedance technique [10], the following relationship is achieved

$$
\frac{\gamma_{t}}{\gamma_{r}}=\sqrt{\frac{G_{t}+j \omega C_{t}}{G_{r}+j \omega C_{r}}}
$$

which directly relates to the measurement values through the following "line-line" equation [11]

$$
\begin{aligned}
\operatorname{Tr}\left[M_{t} \cdot M_{r}^{-1}\right] & =2 \cosh \left(\gamma_{r} \cdot l_{r}\right) \cdot \cosh \left(\gamma_{t} \cdot l_{t}\right) \\
& -\left(\frac{\gamma_{r}}{\gamma_{t}}+\frac{\gamma_{t}}{\gamma_{r}}\right) \cdot \sinh \left(\gamma_{r} \cdot l_{r}\right) \cdot \sinh \left(\gamma_{t} \cdot l_{t}\right),
\end{aligned}
$$

where $\operatorname{Tr}[X]$ denotes the trace operation of matrix $X$. The cascaded matrices $M_{r}$ and $M_{t}$ are transformed from the measured S-parameters of the two CPW lines [8].

If we utilize a bare CPW line as the reference line (i.e., air works as the reference material), its $G_{r}$ is negligible due to the low loss property of the employed substrate, and its $C_{r}$ is available with a two-dimensional (2D) simulation on the crosssection of the bare line [9]. Besides, the bare line's propagation constant $\gamma_{r}$ is readily calculated with (2) by measuring two bare CPW lines that are of clearly distinct lengths [2]. Next, with known $l_{k}$, the propagation constant $\gamma_{k}$ of the $k$ th DUT in Fig. 1 can be determined by using an optimization algorithm on (2), which is followed by the calculation of parameters $C_{k}$ and $G_{k}$ according to (1). Mathematically, when the $k$ th DUT is embedded in the test fixture, with its four parameters and the seven unknown parameters of $S^{X}$ and $S^{Y}$ [1], [4], we can calculate the S-parameters between the two ports of the VNA using the following formula [6]

$$
\begin{aligned}
S^{k c}=F( & S_{12}^{X} S_{12}^{X}, S_{11}^{X}, S_{22}^{X}, S_{21}^{X} S_{12}^{Y}, \\
& \left.S_{12}^{Y} S_{12}^{Y}, S_{11}^{Y}, S_{22}^{Y}, C_{k}, G_{k}, \gamma_{k}, l_{k}\right),
\end{aligned}
$$

where parameters $C_{k}, G_{k}, \gamma_{k}$, and $l_{k}$ are used to calculate the $\mathrm{ABCD}$ matrix first with the following equation [8]. The calculated ABCD matrix is subsequently transformed to $\mathrm{S}$ parameters $S^{k}$, as shown in Fig. 1 .

$$
\left[\begin{array}{ll}
A & B \\
C & D
\end{array}\right]^{k}=\left[\begin{array}{cc}
\cosh \left(\gamma_{k} l_{k}\right) & \frac{\gamma_{k} \cdot \sinh \left(\gamma_{k} l_{k}\right)}{G_{k}+j \omega C_{k}} \\
\frac{\left(G_{k}+j \omega C_{k}\right) \cdot \sinh \left(\gamma_{k} l_{k}\right)}{\gamma_{k}} & \cosh \left(\gamma_{k} l_{k}\right)
\end{array}\right]
$$

If $S^{k m}$ is defined as the measured S-parameters of the test fixture with the $k$ th standard embedded, as shown in Fig. 1, residuals between $S^{k m}$ and the calculated $S^{k c}$ of all $n$ standards can be defined as

$$
\left|S_{e r r}\right|=\sqrt{\sum_{k=1}^{n} \sum_{i, j=1}^{2}\left|S_{i j}^{k m}-S_{i j}^{k c}\right|^{2}}
$$

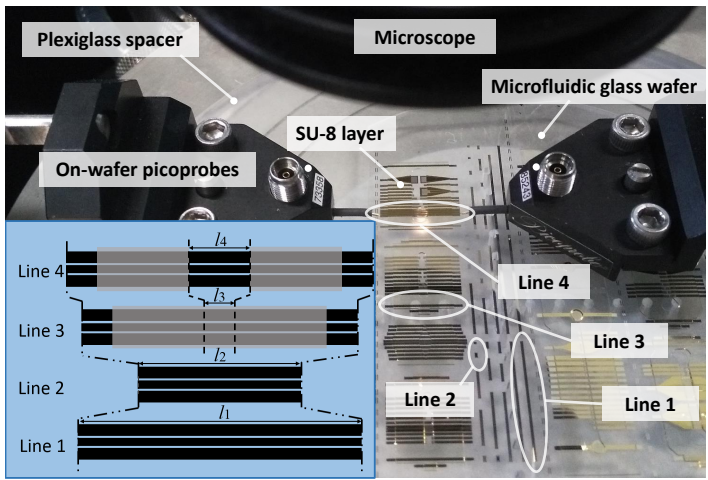

Fig. 2. The measurement setup and devices for multiline multi-material calibration technique, with the inset presenting the schematics of two bare lines ("Line 1" and "Line 2"), an SU-8 line ("Line 3"), and a microfluidic line ("Line 4"), where $l_{1}, l_{2}, l_{3}$, and $l_{4}$ indicate the lengths of interest.

Starting with appropriate initial values, the seven parameters of the two error boxes $S^{X}$ and $S^{Y}$ can be determined by minimizing this equation at every frequency point with a nonlinear least squares method [4], [6].

\section{Measurement Results And Discussion}

Validation measurements were performed on a probe-station connecting an Agilent E8361A VNA, as shown in Fig. 2. Four CPW lines that are patterned on a low loss glass substrate are used, with the cross-section geometry of these lines being $20 \mu \mathrm{m}-100 \mu \mathrm{m}-150 \mu \mathrm{m}$ (gap-signal-ground). Specifically, "Line 1" and "Line 2" are two bare CPW lines whose lengths, i.e., $l_{1}$ and $l_{2}$, are $20.53 \mathrm{~mm}$ and $1 \mathrm{~mm}$, respectively. "Line 3" is an SU-8 loaded line and "Line 4" is a microfluidic calibration fixture, where the SU-8 length $l_{3}$ and channel length $l_{4}$ are $1.5 \mathrm{~mm}$ and $3 \mathrm{~mm}$, respectively.

Several steps were taken for validating the multiline multimaterial calibration technique. First, S-parameters of "Line 1" and "Line 2" were recorded, from which the propagation constant $\gamma_{\text {air }}$ and p.u.l capacitance $C_{\text {air }}$ of an empty CPW line were obtained. Second, S-parameters of the SU-8 line"Line 3" and the microfluidic line-"Line 4" before and after loading deionized (DI) water were recorded, representing three calibration standards. Third, by the combination of (2) and (1), we are able to extract the parameters $\gamma_{S U 8}, C_{S U 8}$, and $G_{S U 8}$ of an SU-8 loaded CPW line and $\gamma_{\text {water }}, C_{\text {water }}$, and

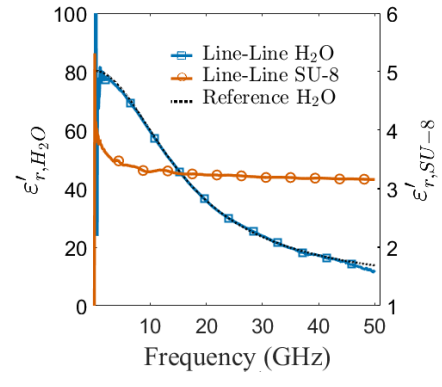

(a)

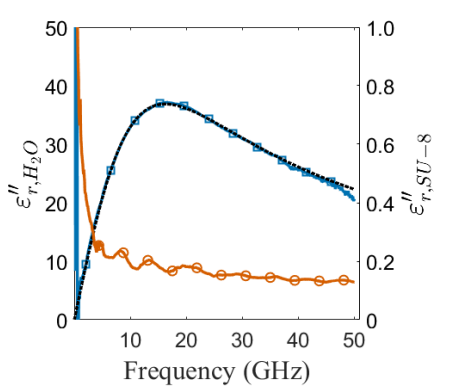

(b)
Fig. 3. Measured complex permittivities of DI water and SU-8 at $19.6^{\circ} \mathrm{C}$. As a reference, literature values of water are also shown [12]. 


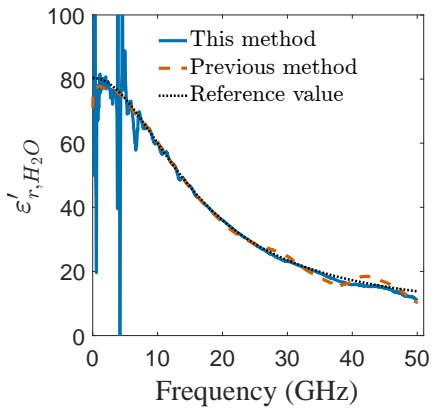

(a)

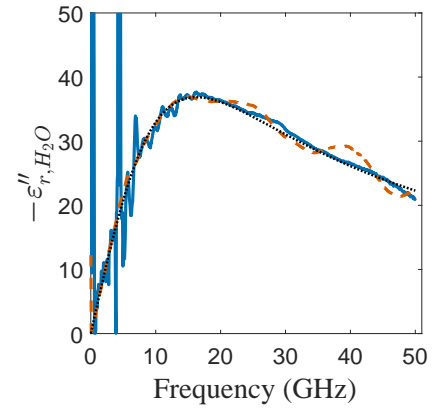

(b)
Fig. 4. Measured DI water permittivities at $19.6^{\circ} \mathrm{C}$, which are extracted from $\mathrm{S}$-parameters that are calibrated with previous two-tier calibration procedure and the proposed calibration technique, respectively.

$G_{\text {water }}$ of a water loaded CPW line. With the capacitances and conductances, complex permittivities of SU-8 and DI water were calculated [9], which are reported in Fig. 3. Finally, with all of the parameters of bare line, SU-8 line, and water line, the two error boxes $S^{X}$ and $S^{Y}$ can be accurately determined with the algorithm described in previous section.

Another line with similar structure to "Line 4" yet of $1.5 \mathrm{~mm}$ microfluidic channel length was used to verify the proposed multiline multi-material calibration method. We first extracted the complex permittivity of DI water from the $\mathrm{S}$-parameters that were calibrated with the multiline multimaterial method, as presented in Fig. 4. As a comparison, Fig. 4 also presents the permittivity results obtained from the $\mathrm{S}$-parameters that were calibrated with the regularly used twotier calibration method [9]. In the two-tier method, a typical first-tier multiline TRL calibration [5] was first performed, whereas the de-embedding error boxes were directly calculated with the distributed p.u.l $R, L, C$, and $G$ of the bare line and SU-8 line [9]. Next, we compared the proposed calibration technique and the previous two-tier calibration method with the comparison algorithm [13]. The comparison result of the two calibration procedures is presented in Fig. 5.

According to the permittivity results shown in Fig. 4, at low frequencies, the proposed calibration technique is worse than the previous method. This is related to the trace algorithm of the "line-line" technique, which is preferably applicable at high frequencies [11]. Notably, at high frequencies, e.g., at frequencies between $20 \mathrm{GHz}$ and $50 \mathrm{GHz}$, the permittivity re-

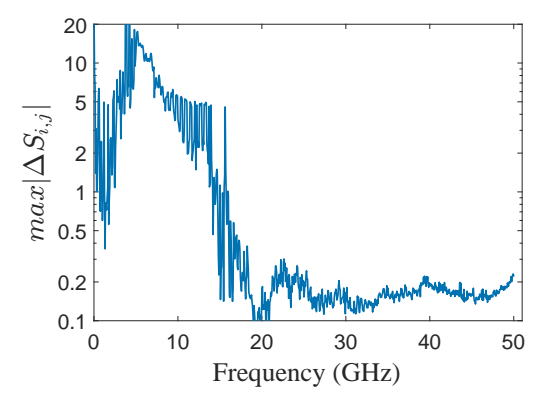

Fig. 5. The comparison between the proposed multiline multi-material calibration technique and the previous two-tier calibration method. sults extracted with the proposed procedure are rather smooth, whereas the permittivities with previous two-tier technique show obvious ripples. The slight comparison difference of the two approaches at frequencies between $20 \mathrm{GHz}$ and 50 $\mathrm{GHz}$ in Fig. 5 partly relates to probe placements, probe contacts, device fabrication tolerance, etc., which are often ignored in previous two-tier calibration process. As a result, the proposed multiline multi-material method can provide reliable calibration, especially at high frequencies. It will be of great advantages in applications where complicated CPW line based devices are employed, e.g., interdigital capacitors.

\section{CONCLUSION}

A multiline multi-material calibration method based on nonlinear least squares algorithm is developed. The combination of a general "line-line" trace theory and an equivalent impedance method forms the foundation of this method. Validation measurements on DI water and the comparison with a previous calibration method have shown its feasibility of accurate calibration at high frequencies. It will be especially useful in applications where more complicated passive devices than transmission lines are used.

\section{REFERENCES}

[1] R. B. Marks, "Formulations of the basic vector network analyzer error model including switch-terms," in 50th ARFTG Conference Digest-Fall, vol. 32. Portland, OR, Dec. 1997, pp. 115-126.

[2] G. F. Engen and C. A. Hoer, “'thru-reflect-line': an improved technique for calibrating the dual six-port automatic network analyzer," IEEE Trans. Microw. Theory Tech., vol. 27, no. 12, pp. 987-993, Dec. 1979.

[3] H.-J. Eul and B. Schiek, "Thru-match-reflect: One result of a rigorous theory for de-embedding and network analyzer calibration," in 18th European Microwave Conference (EuMC). Stockholm, Sweden, Sep. 1988, pp. 909-914.

[4] P. Barmuta, A. Lewandowski, S. Liu, W. Wiatr, and D. Schreurs, "General method of seven-term statistical calibration with partially defined standards," in 20th Microwaves, Radar, and Wireless Communication (MIKON). Gdansk, Poland, Sep. 2014, pp. 1-4.

[5] R. B. Marks, "A multiline method of network analyzer calibration," IEEE Trans. Microw. Theory Tech., vol. 39, no. 7, pp. 1205-1215, July 1991.

[6] D. Williams, "De-embedding and unterminating microwave fixtures with nonlinear least squares," IEEE Trans. Microw. Theory Tech., vol. 38, no. 6, pp. 787-791, Jun. 1990.

[7] S. Liu, I. Ocket, P. Barmuta, T. Markovic, A. Lewandowski, D. Schreurs, and B. Nauwelaers, "Broadband dielectric spectroscopy calibration using calibration liquids with unknown permittivity," in 84th ARFTG Conference Digest-Fall. Boulder, CO, Dec. 2014, pp. 1-5.

[8] R. B. Marks and D. F. Williams, "A general waveguide circuit theory," Journal of Research of the National Institute of Standards and Technology, vol. 97, no. 5, pp. 533-562, Sep.-Oct. 1992.

[9] J. C. Booth, N. D. Orloff, J. Mateu, M. Janezic, M. Rinehart, and J. A. Beall, "Quantitative permittivity measurements of nanoliter liquid volumes in microfluidic channels to $40 \mathrm{GHz}$," IEEE Trans. Instrum. Meas., vol. 59, no. 12, pp. 3279-3288, Dec. 2010.

[10] M. D. Janezic and D. F. Williams, "Permittivity characterization from transmission-line measurement," in 1997 IEEE MTT-S Int. Microwave Symp. Dig., vol. 3. Denver, CO, Jun. 1997, pp. 1343-1346.

[11] X. Bao, S. Liu, I. Ocket, J. Bao, D. Schreurs, S. Zhang, C. Cheng, K. Feng, and B. Nauwelaers, "A general lineline method for dielectric material characterization using conductors with the same cross-sectional geometry," IEEE Microw. Wirel. Compon. Lett., vol. 28, no. 12, pp. 356358, Apr. 2018.

[12] W. J. Ellison, "Permittivity of pure water, at standard atmospheric pressure, over the frequency range $0-25 \mathrm{THz}$ and the temperature range $0-100^{\circ}$ C," J. Phys. Chem. Ref. Data, vol. 36, no. 1, pp. 1-18, Mar. 2007.

[13] D. F. Williams, R. B. Marks, and A. Davidson, "Comparison of on-wafer calibrations," in 38th ARFTG Conference Digest-Winter, vol. 20. San Diego, CA, Dec. 1991, pp. 68-81. 